\title{
Positioning More Than Moore Characterization Needs and Methods within the 2011 ITRS
}

\author{
Mart Graef \\ Delft Institute of Microsystems and Nanoelectronics (Dimes) \\ Delft University of Technology \\ Feldmannweg 17, 2628 CT Delft, The Netherlands
}

\begin{abstract}
The concept "More than Moore" was introduced in the 2005 edition of the International Technology Roadmap for Semiconductors (ITRS), with the purpose to describe technology features that do not fit the miniaturization trends as implied by Moore's Law. These features typically enable non-digital functionalities, such as wireless communication, power management, sensing and actuation. As these new functionalities are progressively being incorporated into system-in-package $(\mathrm{SiP})$ and system-on-chip ( $\mathrm{SoC}$ ) formats, the complexity of the resulting microsystems is rapidly increasing. Consequently, there is a need for new sets of physical parameters and figures of merit for the characterization of emerging technology options in the More than Moore domain. Recently, the International Roadmap Committee (IRC) of the ITRS has proposed a methodology to address this subject.
\end{abstract}

Keywords: Heterogeneous integration, multidisciplinarity, design for reliability, More than Moore, ITRS. PACS: 85.30.-z, 85.40.-e, 85.85.+j.

\section{INTRODUCTION}

The objective of this contribution is to describe the changes that have recently been taking place in the International Technology Roadmap for Semiconductors (ITRS), in particular regarding the extension of the roadmap to include not only the technology trends dominated by feature scaling, but also the new trend towards heterogeneous integration of digital and analog components.

Since its creation in 1998, the focus of the ITRS has been mostly on CMOS, the basic semiconductor technology for memory and logic devices. The roadmap document describes the trends and requirements in the fields of process technology and architecture, design, modeling, testing, etc. [1]. Metrology and characterization methodologies have been developed concurrently with the progress in device engineering. Over time, non-CMOS based technology options, mostly for analog functionalities, have received increasing attention in the ITRS. This is due to the fact that miniaturization and CMOScompatibility of new devices has enabled their integration in multifunctional microsystems, either as monolithic systems-on-chip (SoC), or multicomponent systems-in-package (SiP).

This trend has caused enormous challenges for process technology, system engineering and device

Frontiers of Characterization and Metrology for Nanoelectronics: 2011

AIP Conf. Proc. 1395, 345-350 (2011); doi: 10.1063/1.3657913

(C) 2011 American Institute of Physics 978-0-7354-0965-1/\$30.00 characterization, due to the fact that the complexity of the total system increases, whereas the distinction between the components and the (sub)system tends to blur. The characterization of each individual component may be a challenge in itself, the characterization of the complete microsystem requires not only novel methodologies, but also a new approach.

Some of the implications of these technology trends, as described in the ITRS, will be addressed in this article. Miniaturization, the cornerstone of semiconductor technology development, will be discussed first. Next, a description will be given of the ITRS roadmapping process that is followed by an account on the incorporation of the new 'More than Moore' technology domain into the ITRS. And finally, the consequences of 'More than Moore' for roadmapping and characterization methodologies will be outlined.

\section{MINIATURIZATION}

Miniaturization has been the driving force for the semiconductor industry for more than fifty years, although it has never been a goal in itself. Rather, miniaturization is the basic means to reduce the manufacturing cost while increasing the performance 
(signal processing, data storage) of an electronic device. Moore made the observation in 1965 that that the complexity for minimum component costs has increased at a rate of roughly a factor of two per year. [2] Since then, his original observation became the essence of what has become known as 'Moore's Law': namely, that progress in semiconductor technology development can be characterized as a doubling of the number of components in an integrated circuit every eighteen months

The basic parameter for miniaturization can be either the pitch, i.e. the repetition distance of adjacent pattern features (polysilicon gates or metal lines), or the minimum dimension that can be lithographically reproduced, usually the transistor gate length ( $c f$. Fig. 1). For DRAM devices, the gate length is equal to the half pitch. In general, the half pitch is the best measure to indicate the 'critical dimension' (CD).
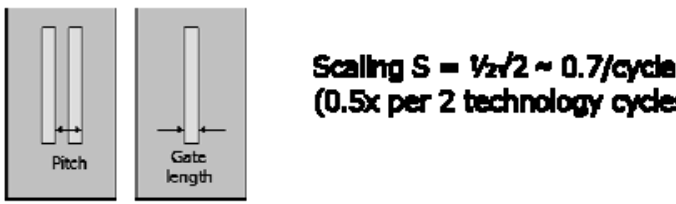
(0.5x per 2 technology cyles)

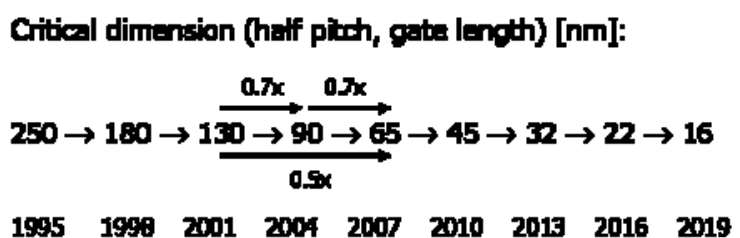

FIGURE 1. CMOS transistor scaling.

The typical scaling factor for every new technology generation is $1 / 2 \sqrt{2}$ or approximately 0.7 . This means that with every technology cycle the total number of transistors on the same surface area can be doubled.

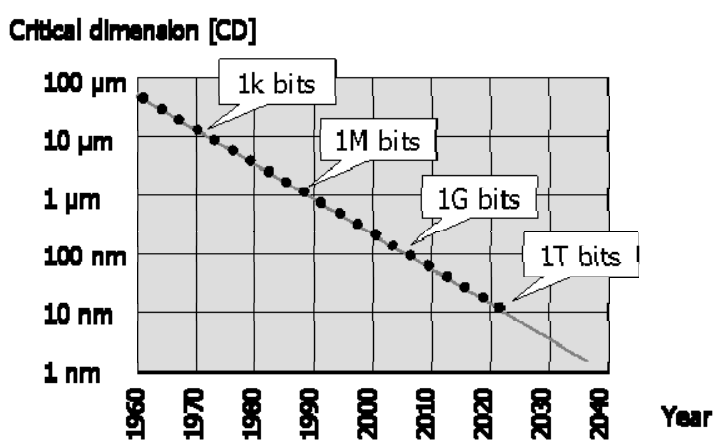

FIGURE 2. Moore's Law: Miniaturization.

This leads to the best-known representation of Moore's Law, viz. a diagram showing an exponential decrease of the critical dimension and an exponential increase of the number of components of an integrated circuit over time (Fig. 2).

Lithography has been the essential enabler for scaling, and consequently for the technology roadmap, by making it possible to reduce the dimensions of the transistors. In due time, signal routing, rather than the size of the transistor, became the limiting factor for integration. This was solved by increasing the number of interconnection levels, which changed the basic concept of the device architecture from a twodimensional into a three-dimensional structure.

The progress in CMOS technology has been the backbone of the ITRS from its inception in 1998 until present. Before addressing the new technology developments that are strongly affecting the roadmapping methodology, it makes sense to spend a few words on the ITRS roadmapping process itself.

\section{THE ITRS ROADMAPPING PROCESS}

Microelectronics has evolved as an industrial branch since about 1963, when the first commercial integrated circuits became available. In these early years, all aspects of research, development and manufacturing of semiconductor devices were considered to be proprietary, essential for the competitive position of the IC supplier. The stages in the supply chain of an IC manufacturing process can roughly be summarized as follows:

- Design and construction of process equipment;

- Development of process recipes;

- Development of process flow (flow-chart) and the implementation of process control (manufacturability);

- $\quad$ Product development.

In the early days, each manufacturer would control the complete value chain, which included even the building of manufacturing equipment. Over the years however, it became clear that only certain stages in the supply chain do have really differentiating value for the IC supplier. Moreover, the development costs for new technologies increased dramatically. This led to a different view on the distinction between precompetitive and proprietary know-how. No single company could afford to sustain the complete value chain on its own. As a consequence, independent semiconductor equipment companies were set up in the 1970s, and a steadily increasing part of the technology supply chain was being outsourced by the IC manufacturers. This trend was extended to process technology itself. In the 1980 s, it was realized that basic semiconductor processes, such as CMOS, could be a generic process, shared by everyone, whereas intellectual property for each IC manufacturer could be 
limited to product development. The sharing of knowhow yielded to the following benefits:

- Reduction of R\&D costs for individual companies;

- Acceleration of R\&D efforts, leading to a shorter time to market;

- Providing guidance for an industrial/academic research agenda.

In the early $1990 \mathrm{~s}$, the need for an industry-wide semiconductor technology roadmap was realized. The Semiconductor Industry Association took the initiative for the first National Technology Roadmap for Semiconductors, which evolved into the ITRS in 1998. The ITRS was established with the objective to provide guidance to the semiconductor industry by predicting technology trends in the industry, spanning 15 years (with more detailed forecasts for the 1-6 years near-term and general trends for 7-15 years longterm). The activities within the scope of the ITRS are supervised by the International Roadmap Committee (IRC). At present, the ITRS has 17 Technology Working Groups (TWGs), addressing the following topics:

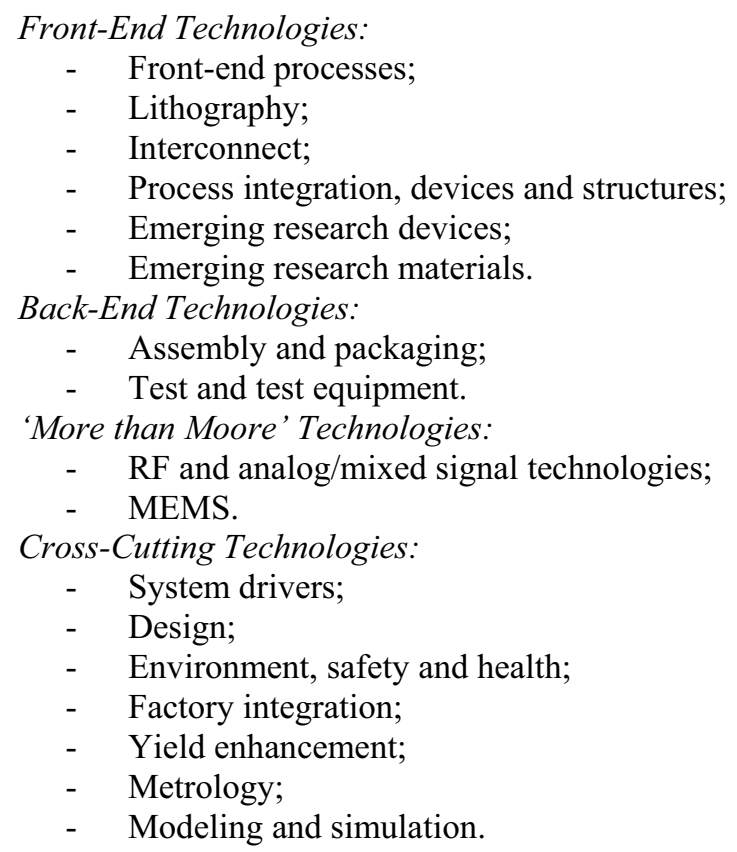

Over 300 participants, representing more than 50 industries, institutes and universities, contribute to the TWGs. A new edition of the ITRS is released in the public domain (www.itrs.net) every two years, odd numbers years, with an update of the tables every other year, even numbered years. It encompasses 17 chapters, over 1000 pages and more than 200 tables. The size, detail and comprehensiveness make this roadmap unique for any industry.

\section{NEW TERRITORY: MORE THAN MOORE}

\section{When will the roadmap end?}

The projection of an exponential trend is an uncertain affair. From the time of its inception, it was anticipated that the semiconductor technology roadmap would end at a certain time, dictated by the constraints of process technology (especially the resolution limitations of lithography) or physical barriers (device sizes approaching atomic dimensions), or economic constraints. At any point in time since the 1970s, the general expectation has been that the technology roadmap would be sustainable for about ten more years. This is still the shared opinion today. A major part of the efforts in the ITRS Technology Working Groups is related to the identification of technology 'roadblocks' that would hamper achieving the projected technology requirements (e.g. CD control and overlay in lithography). Jointly, these roadblocks are known as the 'Red Brick Wall'.

It has become clear that the 'end of the roadmap' is a shifting date. It is being postponed by innovations in process technology (e.g. 193nm / immersion lithography and EUV lithography) and novel device architectures (based upon the idea of 'equivalent scaling', which refers to the extension of geometrical constant field - scaling by new design options and new materials which affect the electrical performance of a device). In addition, new switching device concepts (based upon e.g. quantum effects, carbon nanotubes, molecular electronics) are being pursued in a number of research programs that jointly can be labeled as 'Beyond CMOS'.

The history of the ITRS can be described as a mapping of the itinerary for the fulfillment of Moore's Law. However, over the years it has become clear that transistor scaling is only one of the possible ways to sustain technology development in microelectronics. Other types of electrical (e.g. RF) and non-electrical (e.g. MEMS) types of components could be integrated with the 'classical' IC into compact microsystems. This trend has become known as 'More than Moore'.

\section{More than Moore}

The concept of 'More than Moore' was introduced in the 2005 edition of the ITRS [3]. Soon, it was realized that this was a departure from the idea of semiconductor technology roadmapping based upon miniaturization of transistors only. It put the ITRS 'at the crossroads' [4]. 
There are several ways to describe the 'More than Moore' concept. According to the ITRS definition, the 'More than Moore' approach typically allows for the non-digital functionalities (e.g., RF communication, power control, passive components, sensors, actuators) to migrate from the system board level into a particular package-level (SiP) or chip-level (SoC) implementation. The objective of 'More than Moore' is to incorporate digital and non-digital functionality into compact systems. [1]. A schematic representation is depicted in Fig. 3).

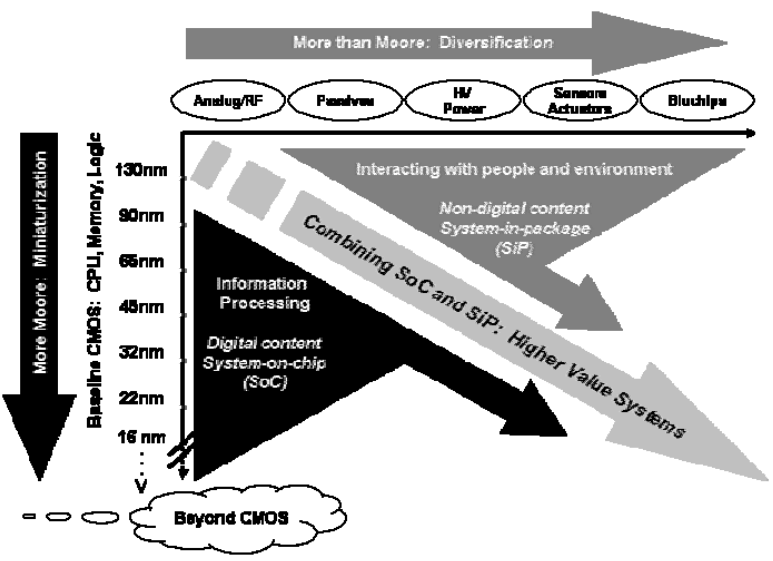

FIGURE 3. A roadmap perspective: 'More Moore' and 'More than Moore'. (Source: ITRS 2011)

The diagram shows that the 'More Moore' trend is driven by miniaturization and focused on the digital content of the device. Information processing is the major functionality. The 'More than Moore' trend is driven by functional diversification, addressing specifically the non-digital content of a microsystem. It should be emphasized that 'More than Moore' does not constitute an alternative to 'More Moore'. The added value is rather to be found in the combination of these two trends, which enables the creation of highly sophisticated multifunctional smart microsystems.

This view is depicted in Fig. 4, which shows that a microsystem can be conceived as an integrated system consisting of digital 'More Moore' and non-digital 'More than Moore' subsystems. The construction of a microsystem from components is not trivial. The 'glue' is provided by a number of disciplines, ranging from system design and materials science to assembly and packaging. Jointly, these enable what may be called 'heterogeneous integration'.

Traditionally, the 'More Moore' trend has been technology-driven: the increase of digital performance and data storage capacity is a measure of progress for microprocessors, memory and logic devices. This is less straightforward for 'More than Moore', which is by nature application-driven (Fig. 5).

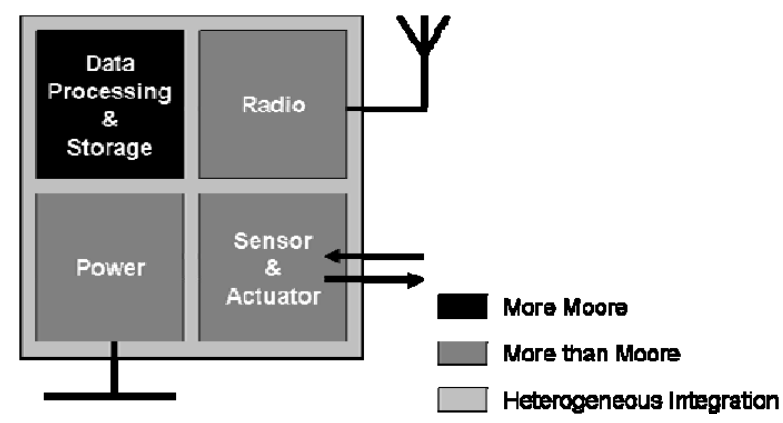

FIGURE 4. A microsystem can be represented schematically as an integrated structure consisting of 'More Moore' (digital) and 'Moore than Moore' (analog) components.

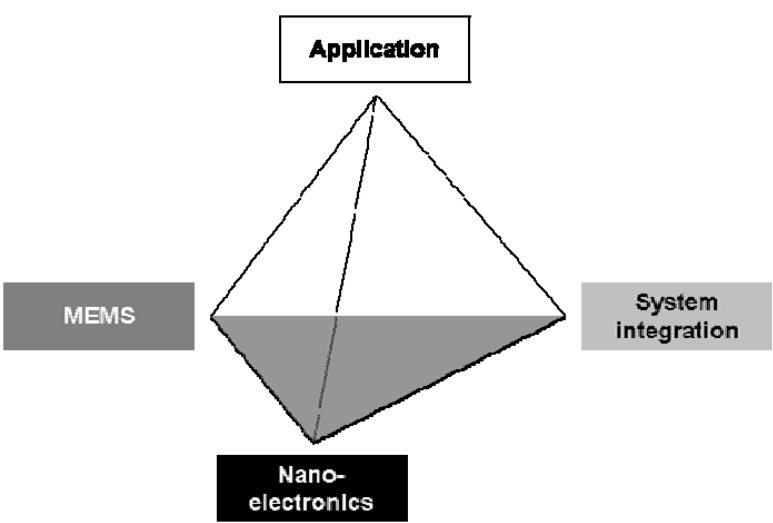

FIGURE 5. 'More Moore' and 'Moore than Moore': Technology-application framework.

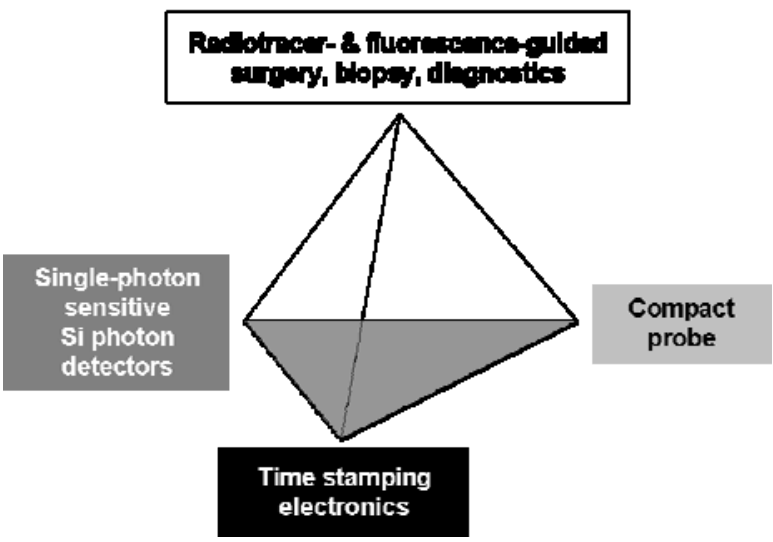

FIGURE 6. An example of the "More Moore' - 'More than Moore' technology-application framework in the healthcare domain. (Courtesy Edoardo Charbon, Delft University of Technology)

The 'technology base' derives from different disciplines. The 'More Moore' part may consist of nanoelectronic components, the 'More than Moore' part may be an integrated MEMS-based sensor or 
actuator. System integration defines the physical implementation of the microsystem.

It is clear that it will be very difficult to define parameters that can describe the 'More than Moore' domain in a generic way, similar to, say, the critical gate length or the threshold voltage in the CMOS domain. For 'More than Moore', the relevant parameters are determined by the intended functionality, or rather the application of the device. An example is given in Fig. 6, which shows an application of 'More than Moore'-based technology in the healthcare domain. The objective here is the creation of probes for the detection of radiotracers used to mark lymph and skin cancer cells, to be used during surgery. This typically multidisciplinary project requires the integration of time-stamping electronics and single-photon detectors into a single system, i.e. a compact probe.

\section{CHARACTERIZING MORE THAN MOORE}

\section{More than Moore methodology}

The ITRS has demonstrated its value for the roadmapping of the CMOS technology domain. In the course of this process, the relevant pre-competitive research domains have been identified, enabling and stimulating cooperation between industries, institutes and universities. This has resulted in the sharing of R\&D efforts, a more efficient use of resources through focus on the major technology challenges and the reduction of development costs and time. At the same time, the ITRS work has facilitated a synchronization of the equipment and materials community with the semiconductor manufacturing community.

The generation of a technology roadmap for the 'More than Moore' domain would offer similar benefits, but is a lot more challenging. Whereas a limited number of clearly identified technical parameters serve as a measure for progress in CMOS, this is not the case for 'More than Moore'. Also, since
'More than Moore' technologies are clearly application-driven, it is more difficult to identify generic processes which could serve, similar to CMOS, a wide range of applications. In order to address this issue, the ITRS has released a 'White Paper', which provides a methodology for the roadmapping of the 'More than Moore' technology domain [5].

The necessary conditions for an industry-wide technical roadmap effort have been identified as follows:

- There needs to be a restricted set of figures of merit (FOM), similar to feature size, clock frequency, etc.

- There must be a convergence of opinions among a majority of the key players on the progress trends that these figures of merit are expected to follow. This may be called a 'law of expected progress' (LEP).

- There should be a sufficient willingness to share technology know-how (SHR).

- There should be a potential market of significant size to justify the efforts for the creation of a roadmap. The applicability of the technology has to be sufficiently wide (WAT).

- There has to exist a community of players (ECO) who can benefit from sharing their technology specifications and requirements.

A preliminary assessment of the perspective for roadmapping of some typical 'More than Moore' subdomains has been indicated in Table 1. In some cases, such as for RF and MEMS (sensors and actuators), roadmapping activities have been incorporated into the ITRS program. For power and imaging technologies, roadmapping efforts are as yet in an early stage. The benefits for roadmapping in the biochips domain are still rather uncertain at this time. It is expected that the list will be extended to include other technology areas in the near future.

'More than Moore' roadmapping is as yet in an early stage. The methodology starts at the application side, where product requirements have to be translated into functions, which in turn define the required device characteristics. This is shown schematically in Fig. 7.

TABLE 1. 'More than Moore' Technology Assessment. (Source: ITRS 'More than Moore' White Paper, 2010)

\begin{tabular}{|c|c|c|c|c|c|c|}
\hline & $\begin{array}{c}\text { FOM } \\
\text { Figure of } \\
\text { merit }\end{array}$ & $\begin{array}{c}\text { LEP } \\
\text { Law of } \\
\text { expected } \\
\text { progress }\end{array}$ & $\begin{array}{c}\text { SHR } \\
\text { Willingness to } \\
\text { share }\end{array}$ & $\begin{array}{c}\text { WAT } \\
\text { Wide } \\
\text { applicability }\end{array}$ & $\begin{array}{c}\text { ECO } \\
\text { Existing } \\
\text { community }\end{array}$ & $\begin{array}{c}\text { In ITRS } \\
\text { today? }\end{array}$ \\
\hline RF & ++ & ++ & + & ++ & + & Yes \\
\hline Power & + & + & $?$ & $=$ & $?$ & No \\
\hline Imaging & + & + & $?$ & $=$ & $?$ & No \\
\hline MEMS & - & $?$ & $?$ & ++ & - & Yes \\
\hline Biochips & - & $?$ & - & $?$ & - & No \\
\hline
\end{tabular}




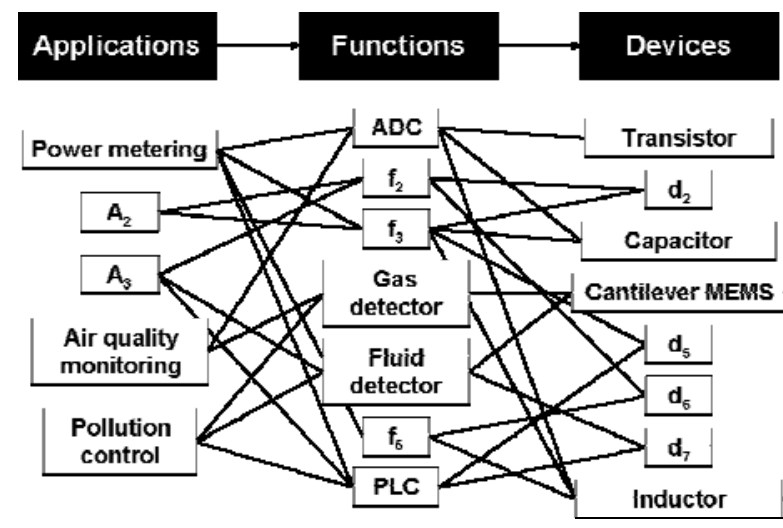

FIGURE 7. 'More than Moore' roadmapping methodology: From applications to functions to devices. (Source: ITRS 'More than Moore' White Paper, 2010)

The objective of the roadmapping methodology is to derive generic technologies, suitable for multiple application areas. This makes it possible to define the key parameters that can be used as figures of merit for the monitoring of technology development.

\section{Characterization of More than Moore devices}

A More than Moore-based microsystem has the following characteristics:

- It is a system-in-package, with digital and nondigital functions (RF, passives, sensors, fluidics, optics, etc.) integrated in one module. The package is a functional component.

- It incorporates a high diversity of technologies. This implies that modular technology platforms have to be developed in a structured design environment.

- It is compatible with a CMOS-based process, which enables the integration of non-digital components with digital functionality.

It is evident that the complexity of these systems constitutes a major challenge for characterization and testing. This may be illustrated by the following key terms:

\section{Multidisciplinarity}

The multifunctionality of the microsystem requires not only methodologies from electrical engineering, but also from other disciplines, such as (micro)mechanics, materials science, thermomechanics, optics, chemistry, biology, medical science and many more.

\section{Heterogeneity}

A microsystem is typically composed from a number of sub-systems, designed and assembled to operate jointly. Whereas characterization methodologies for each of the subsystems may be available, this is in general not the case for the complete system, incorporating 3D structures interconnected by throughsilicon vias, new materials interfaces, etc.

\section{Complexity}

The merging of subsystems creates a new 'middle end', with new requirements for characterization, which have to cover multiple scales (from nanometer to millimeter scale).

\section{Reliability}

The typical heterogeneity and complexity of smart microsystems requires the development of new methods for reliability assessment.

\section{CONCLUSION}

The transition from 'microelectronics' to 'nanoelectronics' may be formally defined as the point in time when the minimum feature size in state-of-theart ICs became smaller than $100 \mathrm{~nm}$. At about the same time, however, around 2005, the miniaturization trend ('More Moore') was extended with a multifunctional integration trend labeled 'More than Moore'.

Since then, the ITRS has taken up the task to define and characterize the emerging 'More than Moore' technology requirements. A methodology for the roadmapping of this domain has been developed. Characterization methodologies for 'More than Moore' devices are in high need. The key terms to describe the distinctive 'More than Moore' features in this regard are multidisciplinarity, heterogeneity, complexity and reliability.

\section{ACKNOWLEDGMENTS}

The author gratefully acknowledges the 'More than Moore' related efforts by his colleagues in the International Roadmap Committee of the ITRS and the CATRENE Working Group on 'More than Moore', in particular Michel Brillouët, Patrick Cogez, Bert Huizing and Reinhard Mahnkopf.

\section{REFERENCES}

1. International Technology Roadmap for Semiconductors 2009 Edition and 2010 Update, www.itrs.net.

2. G. Moore, Electronics 38, nr. 8 (April 19, 1965).

3. International technology Roadmap for Semiconductors 2005 Edition - Executive Summary, www.itrs.net.

4. W. Arden, P. Cogez, M. Graef, Nikkei Microdevices nr. 270 (December 2007), 138

5. 'More-than-Moore' White Paper (2010), www.itrs.net. 